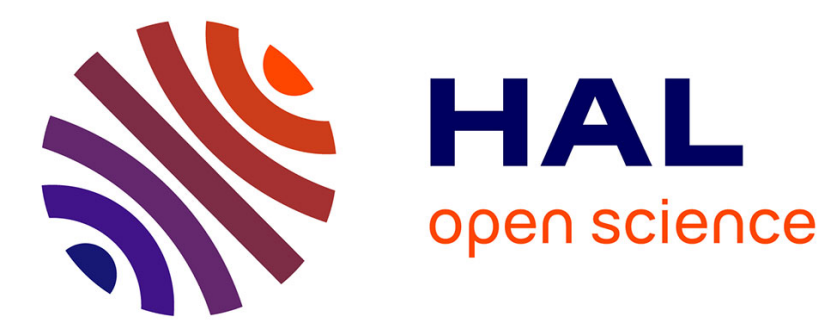

\title{
INTRODUCTION : REPENSER L'IMPACT DE L'ADHESION
}

Dorota Dakowska, Laure Neumayer

\section{To cite this version:}

Dorota Dakowska, Laure Neumayer. INTRODUCTION : REPENSER L'IMPACT DE L'ADHESION.

Politique européenne, 2005, p.5-27. halshs-00963730

\section{HAL Id: halshs-00963730 \\ https://shs.hal.science/halshs-00963730}

Submitted on 21 Mar 2014

HAL is a multi-disciplinary open access archive for the deposit and dissemination of scientific research documents, whether they are published or not. The documents may come from teaching and research institutions in France or abroad, or from public or private research centers.
L'archive ouverte pluridisciplinaire HAL, est destinée au dépôt et à la diffusion de documents scientifiques de niveau recherche, publiés ou non, émanant des établissements d'enseignement et de recherche français ou étrangers, des laboratoires publics ou privés. 


\section{Dorota DaKowska}

Laure NEUMAYER

\section{INTRODUCTION : REPENSER L'IMPACT DE L'ADHESION}

L'adhésion à l'Union européenne des dix nouveaux pays membres issus d'Europe centrale et orientale et de la Méditerranée, survenue le 1er mai 2004, invite à un approfondissement des recherches menées sur les réformes politiques et institutionnelles effectuées dans ces pays sous l'influence du facteur européen, en les associant étroitement à la réflexion sur l'intégration européenne.

La clôture des négociations d'adhésion et l'accélération de la reprise de l'acquis dans les derniers mois précédant l'adhésion marquent une étape finale dans le rapprochement de ces pays avec les structures communautaires. L'entrée dans l'Union européenne constitue une échéance essentielle sur le plan politique, symbolique et de mise en œuvre de politiques publiques. Les nouveaux membres doivent désormais prouver leur capacité à s'intégrer au système de gouvernance multi-niveaux communautaire et à répondre aux exigences que celui-ci implique au niveau interne. Il devient aussi possible de s'interroger sur les conséquences de l'élargissement, considéré comme un processus étendu dans sa durée, à la fois sur le plan des transformations des systèmes politiques des nouveaux Etats membres et sur celui du développement de l'UE élargie. En effet, l'adhésion signifie un changement qualitatif dans les rapports entre les acteurs européens « anciens » et " nouveaux », avec l'accroissement du poids de ces derniers dans le processus de prise de décision et le cadre institutionnel de l'Union. Alors que l'influence des pays candidats se traduisait auparavant par la mise en avant d'arguments fondés sur des pressions sociales et politiques internes (par exemple lors des négociations sur les périodes transitoires) ou par une participation timide aux forums auxquels ils étaient conviés, ils codécident désormais du fonctionnement de l'Union.

Ce basculement fonde la nécessité d'un renouvellement épistémologique des études de l'Union européenne élargie, en développant des approches moins centrées sur le caractère asymétrique des relations entre les anciens et les nouveaux pays membres, et plus tournées vers leurs interdépendances dans un système de prise de décision à niveaux multiples. Du fait de la Politique européenne, $\mathrm{n}^{\circ} 15,2005$, p.5-27 
focalisation de l'étude de la pré-adhésion sur l'UE et ses Etats membres, trois "points aveugles» apparaissent en effet dans une grande partie de la littérature sur l'élargissement à l'Est :

1. Les singularités et les limites de la reprise de l'acquis communautaire dans les Etats candidats, qui sous-tendent l'hypothèse d'une différenciation entre pays et entre secteurs de politique publique. Des analyses comparatives diachroniques permettraient de mettre en lumière les dynamiques et les obstacles à l'inclusion dans le système existant de gouvernance multi-niveaux depuis le début des années 1990.

2. La variété des acteurs gérant l'élargissement (du côté communautaire) et la pré-adhésion (du côté des pays candidats), qui sont impliqués dans le système communautaire de régulation politique et économique multi-niveaux. Il semble important d'élargir le champ de l'analyse pour y inclure les acteurs non institutionnels, infra-nationaux et trans-nationaux (groupes d'intérêt, partis politiques, associations, collectivités locales) et ainsi avoir une vision plus complète des interactions sous-tendant l'élargissement.

3. L'anticipation de l'adhésion, avec la formation de nouvelles élites administratives et partisanes, la création de groupements transnationaux et la mobilisation de différents acteurs politiques et sociaux, apparait cruciale. Comment les acteurs des futurs Etats membres tentent-ils de faire entendre leur voix dans les arènes européennes et d'y acquérir des ressources politiques? Comment redéfinissent-ils leurs stratégies et leurs valeurs dans ce nouvel environnement ? Dans quelle mesure la perspective d'adhésion à l'UE est-elle une opportunité et une contrainte, susceptible d'usages différents au niveau national et dans des interactions croissantes avec les institutions communautaires?

Ce dossier propose un retour - épistémologique mais également empirique - sur les enjeux et les conséquences du processus d'élargissement et de l'adhésion elle-même. Par opposition à de nombreux travaux qui mettent en avant son caractère inédit, et sans nier son impact majeur sur l'avenir de l'intégration européenne, les articles présentés ici plaident pour une «normalisation » de l'étude de l'élargissement autour de deux axes : la reprise des outils conceptuels usuels des études européennes et un intérêt marqué pour les acteurs. 
Il s'agit d'appliquer à l'objet «élargissement de l'UE» les approches de l'espace politique européen sous l'angle de l'emboîtement de différents niveaux de prise de décision (micro, méso, macro-politique) dans trois types d'arènes (infra-nationales, nationales et supranationales). Par ailleurs l'approche sociologique, qui a ouvert de nouvelles pistes dans les recherches européennes, apparait utile pour relativiser la césure de l'adhésion. En analysant l'élargissement à travers les configurations d'acteurs qui se sont constituées depuis le début des années quatre-vingt-dix, celui-ci apparait moins comme une rupture que comme une échéance qui scelle de jure des changements ayant eu lieu de facto. Une telle perspective nuance le modèle couramment utilisé, mais souvent réducteur, de la conditionnalité et de «l'exportation» de la gouvernance communautaire dans les nouveaux Etats membres.

\section{LES ETUDES EUROPEENNES FACE AUX SPECIFICITES DE L'ELARGISSEMENT A L'EST}

La pré-adhésion a donné lieu à une multitude de travaux qui tendent le plus souvent à rendre compte des étapes de rapprochement entre les pays d'Europe centrale et les institutions communautaires d'une façon descriptive, voire prescriptive. Ces écrits, qui répondaient à un besoin d'information sur un élargissement sans précédent dans son étendue et fournissaient une synthèse utile des événements en cours, apportent rarement une contribution à la problématisation plus poussée de l'objet. La complexité avérée de la préparation à l'adhésion, les exigences formulées à l'encontre des pays candidats face au poids des héritages communistes, les instruments de contrôle forgés par la Commission, et enfin l'impact anticipé de l'adhésion sur le fonctionnement de l'Union européenne, renforcent la perception de l'élargissement comme un processus exceptionnel (Mayhew, 1998). Or cette perception a des conséquences importantes sur les outils analytiques privilégiés. Les analyses qui se rattachent aux études européennes, comme les travaux qui s'inscrivent dans la recherche sur le "postcommunisme», négligent bien souvent les phénomènes de réappropriation des normes communautaires dans ces pays. 


\section{Des analyses marquées par l'empirie et la normativité}

Les conditions de production des premiers travaux sur l'élargissement ont déterminé leur fort empirisme, et leur caractère souvent normatif. L'élargissement a constitué un processus fortement médiatisé, car l'UE mettait l'accent sur sa progression pour gérer ses relations avec des pays ayant exprimé dès 1990 le souhait d'adhérer à ses structures. Il a suscité une tentation bien connue des spécialistes de l'Europe de l'Est au moment de la chute du mur de Berlin : celle de s'ériger en « conseillers du prince » (Santiso, 1996).

Les fondations et les centres de recherche appliquée ont joué un rôle majeur dans la rédaction d'ouvrages parfois co-financés par la Direction des Etudes de la Commission européenne ou d'autres institutions internationales comme la Banque mondiale ou l'OCDE. Ceux-ci reposaient sur la collaboration de chercheurs, de fonctionnaires nationaux ou communautaires, et d'experts des Etats membres ou des Etats candidats, se situant sur un registre mipolitique mi-universitaire. Deux éléments caractérisent ce type d'études : une prééminence des analyses économiques sous couvert d'interdisciplinarité, ainsi qu'une approche prospective et des recommandations aux acteurs gouvernementaux - sous forme d'appels à l'accélération de l'élargissement et à la réforme interne de l'Union européenne, ou de critique des critères d'adhésion (Pinder, 1991). Les thèmes majeurs d'un grand nombre d'ouvrages sont les suivants : un rappel de la politique orientale de l'Union européenne, une présentation des implications de l'élargissement pour certaines politiques communautaires, et un aperçu de l'état des réformes des pays candidats (Mannin, 1999, Ingham et Ingham, 2002, Hausner et Marody, 2000). Certaines nuances sont apportées à ce schéma général, avec un accent mis sur la prospective sous forme de scénarios d'élargissement (Maresceau, 1997), sur les leçons à tirer des élargissements précédents (Michalski et Wallace, 1992), ou encore sur le «dilemme » entre approfondissement et élargissement (Grabbe et Hughes, 1997).

Ces analyses prospectives s'accompagnent d'études descriptives cherchant à évaluer, à l'aide d'outils plus ou moins sophistiqués, le degré de préparation des pays candidats à l'adhésion. La fondation Bertelsmann a ainsi lancé une série d'ouvrages comparatifs, selon une 
grille d'analyse directement calquée sur les évaluations de la Commission européenne (Weidenfeld, 1996). La compréhension des dynamiques de la pré-adhésion est des plus limitées dans ce type d'études : leurs coordinateurs se bornent à souligner les corrélations positives entre réformes postcommunistes et adhésion, mais aussi le fait qu'aucun des pays étudiés ne remplit les conditions d'adhésion et qu'il leur faut poursuivre leurs efforts dans ce sens. Des travaux ayant un objectif comparable sont parallèlement réalisés dans les pays candidats tout au long de la pré-adhésion (Chorośnicki, Cziomer, 1997, Inotai, 2001, Šmejkal, Had, Polášková, 1998).

Ces analyses ont un biais normatif implicite, car les opposants à certaines réformes y sont présentés comme des obstacles au processus d'intégration et donc de modernisation - sans que soit discuté le postulat d'un impact positif des exigences de l'UE à l'égard des pays candidats. Elles n'échappent pas non plus aux pièges du déterminisme. La reprise de l'acquis est évaluée à travers l'adoption de lois et la création de nouvelles structures institutionnelles, sans s'interroger sur la mise en œuvre des textes adoptés ou sur le poids réel des institutions créées sous l'impulsion communautaire. La stabilité des systèmes politiques et des indicateurs macroéconomiques sont les critères les plus fréquemment utilisés pour mesurer la «convergence» entre Etats membres et Etats candidats, dans des études extrêmement informatives et détaillées, mais rapidement datées.

\section{Le mésusage des concepts dans les études de l'élargissement}

Deux concepts développés pour étudier le caractère polycentrique et sui generis de la prise de décision dans l'Union européenne, à savoir la « gouvernance » et «l'européanisation », sont partiellement détournés de leur acception initiale dans certaines approches du processus d'élargissement (Dakowska, 2003).

La politique orientale communautaire est qualifiée dans plusieurs travaux de "gouvernance de l'élargissement». Une lecture sous l'angle de l'asymétrie et de la conditionnalité la présente comme dominée par des acteurs bureaucratiques et gouvernementaux, et structurée sur un mode vertical plutôt qu'horizontal (Schimmelfennig, 
Engert, Knobel, 2002, Dimitrova, 2002). Optant pour une perspective unilatérale et top-down, cette approche exclut d'emblée le canal non-gouvernemental ou méso-politique des interactions politiques. Elle réduit les relations entre l'UE et les pays candidats à un processus de fabrication de l'adaptation (generating compliance) et sous-estime les héritages nationaux en amplifiant la convergence institutionnelle entre les pays postcommunistes. Un des biais de ces approches en termes de conditionnalité consiste à considérer les enjeux électoraux et les structures sociales de ces pays comme marginaux dans le changement institutionnel et l'adoption des normes européennes, au motif de la faiblesse de la société civile et des partis politiques d'Europe centrale (Schimmelfennig, Engert, Knobel, $2002: 8)$.

Ce refus de prendre en compte les mobilisations des acteurs des futurs Etats membres et leurs capacités de réponse, voire de résistance aux pressions européennes, témoigne d'une méconnaissance des scènes politiques internes - mais aussi de la négligence des travaux sur l'européanisation qui font des éléments de veto au niveau national (veto-points) l'une des variables déterminant les formes et l'issue du processus de l'européanisation (Risse, Börzel, 2000). Si la dimension coercitive et le rôle directeur de la Commission européenne sont saillants dans la politique d'élargissement (Robert, 2001), la focalisation exclusive sur le côté communautaire n'est pas dépourvue de simplifications. En se concentrant sur les acteurs bureaucratiques et sur les solutions juridiques destinées à répondre aux pressions à l'adaptation, on sous-estime systématiquement les freins structurels et conjoncturels à la mise en œuvre de l'acquis communautaire. Ce faisant l'aspect interactionnel, le poids des caractéristiques institutionnelles locales et par conséquent la diversité de l'impact de l'Union européenne sur les structures politiques sont négligés.

Par ailleurs, les premiers usages de la notion « d'européanisation » dans le cas des pays d'Europe centrale sont vagues et souvent normatifs. Dans un sens téléologique, on assimile le rapprochement des pays postcommunistes avec les structures communautaires au « retour à l'Europe », en considérant la reprise des liens avec l'Europe occidentale comme une question avant tout identitaire, émotionnelle 
et symbolique (Skotnicka-Illasiewicz, 1993, Batt, 1997). Sur ce point, ces travaux n'échappent pas aux mésusages du concept d'européanisation mis en évidence par Claudio Radaelli : cette notion est utilisée pêle-mêle pour signifier l'importance croissante des questions européennes au niveau national, l'intégration européenne, la convergence, ou encore la modernisation (Radaelli, 2001). L'européanisation est ainsi présentée comme un processus, un outil pour la réforme et un objectif de politique publique pour les pays candidats (Ágh, 1999). Ces analyses, qui considèrent la pré-adhésion sous l'angle des ajustements permettant l'intégration à l'UE, s'inscrivent implicitement dans la thématique du "rattrapage» des pays d'Europe centrale. Ils postulent une équivalence entre réformes postcommunistes et pré-adhésion et voient dans l'entrée dans l'UE la seule finalité légitime des transformations postcommunistes (Neumayer, 2004).

On est face à un paradoxe. Dans les études portant sur les Etats membres de l'UE, les approches par la gouvernance et l'européanisation visent à interpréter les divergences persistantes des systèmes politiques nationaux, en dégageant les influences mutuelles entre dynamiques politiques internes et pressions communautaires (Green Cowles et al, 2001, Héritier, 2001, Lequesne, Bulmer, 2002, Kohler-Koch, Eising, 2000). Dans les pays candidats, l'usage souvent peu réflexif, voire abusif de ces concepts induit au contraire l'idée d'une diffusion linéaire des normes communautaire. Implicitement, les hypothèses relatives à l'impact différencié de l'intégration européenne sur les structures nationales sont considérées comme réservées aux Etats membres de l'UE.

\section{LE RENOUVELLEMENT DES ANALYSES DE L'ELARGISSEMENT}

La fin des années quatre-vingt-dix a vu l'apparition de travaux plus analytiques et rigoureux sur l'élargissement de l'Union européenne (Saurugger, 2001). D’une part, ces analyses bénéficient de l'enrichissement des débats théoriques sur l'intégration européenne. D'autre part, l'imbrication croissante entre les réformes communautaires internes et la perspective d'adhésion des nouveaux membres favorise une utilisation plus nuancée des outils conceptuels 
développés pour l'étude du cadre communautaire. Ces questionnements, riches sur le plan théorique, adoptent toutefois une vue dans l'ensemble surplombante : ils se focalisent souvent sur les acteurs institutionnels les plus visibles de l'élargissement, à savoir la Commission européenne et les gouvernements nationaux, pour expliquer les grandes décisions qui rythment la pré-adhésion lors des sommets européens.

\section{Une réflexion approfondie en termes d'européanisation et de gouvernance}

Certaines interrogations en termes « d'européanisation » prennent en compte l'anticipation de l'adhésion dans les pays candidats et la façon dont la contrainte européenne est intériorisée au niveau national (Linden, 2003). De rares travaux se penchent sur la mobilisation d'acteurs intermédiaires des Etats membres ou candidats à l'adhésion (Fink-Hafner 1997, Perez-Solorzano Borragan 2001). D'autres études portant sur les administrations nationales présentent la perspective d'adhésion comme une «incitation puissante à la modernisation de l'Etat", et considèrent l'européanisation comme une anticipation de l'adhésion, incrémentale et différenciée (Lippert, Umbach, Wessels, 2001, Goetz, 2001). L'intérêt de ces études est de mettre en évidence d'une part, la dimension interactive de la relation entre le système communautaire et les acteurs des pays candidats, et d'autre part, les tensions suscitées par cette adaptation. Mais parce qu'elles se placent au niveau macro-politique, celui des structures institutionnelles, sans descendre au niveau méso-politique pour examiner l'élaboration et la mise en œuvre des politiques publiques, elles laissent dans l'ombre les interactions des acteurs politiques et sociaux d'Europe centrale.

Heather Grabbe offre toutefois une analyse nuancée de l'européanisation en matière institutionnelle (Grabbe, 2001). Le rythme de l'ajustement aux normes communautaires, la réceptivité des élites centre-européennes aux modèles extérieurs et l'étendue de l'agenda d'adhésion semblaient prédire une convergence accrue des structures des pays candidats. Or les pressions à l'adaptation exercées par l'Union européenne sont freinées par deux facteurs. Le premier est l'impact diffus de l'influence communautaire, dû à l'absence de 
modèle institutionnel unique, aux tensions intrinsèques aux agendas européens ainsi qu'à la complexité des constellations d'acteurs. Cette complexité alimente l'incertitude quant aux dates, aux standards à atteindre et à l'étendue des réformes requises pour l'entrée dans l'UE. Les dynamiques politiques internes et externes constituent un second facteur de résistance à l'influence européenne: les acteurs ne se soumettent pas passivement aux demandes communautaires, ils se mobilisent sur les enjeux européens.

Ainsi, les pressions de l'Union européenne comportent des tensions internes qui résultent des affrontements entre différents acteurs sectoriels et politiques (Torreblanca 1998, Sedelmeier 2001, 2002). Leur réception dans les pays candidats ne va pas non plus sans heurts. Une étude de la reprise de l'acquis communautaire en matière de réglementation des aides d'Etat en République tchèque et en Pologne montre par exemple le caractère diffus et inégal de l'européanisation, du fait des mobilisations concurrentes des acteurs nationaux. En dépit de la création d'institutions de protection de la concurrence dès le début des années 1990, il faut attendre l'ouverture des négociations d'adhésion en 1999 pour que ces institutions soient dotées des moyens juridiques leur permettant de faire respecter les normes communautaires en matière d'aides publiques. Des conflits sur la légitimité de l'acquis communautaire et sur la nécessité de ce type d'aides dans des économies en transformation continuent cependant à opposer les acteurs politiques et économiques (Neumayer, 2003).

Les exigences communautaires possèdent également des incohérences internes qui peuvent aboutir à des résultats contraires aux déclarations initiales dans des domaines clés, en créant des effets pervers au niveau national. En ce qui concerne les relations entre le pouvoir législatif et l'exécutif, la marginalisation des parlements nationaux fait partie de ces "effets secondaires» (Agh, 1999). Le «biais administratif» consiste en l'émergence d'un noyau dur, un «îlot d'excellence» au sein de l'exécutif, sans que les compétences européennes se diffusent dans l'ensemble des administrations (Lippert, Umbach, Wessels, 2001). Dans les relations centrepériphérie, la création de nouvelles autorités régionales peut s'accompagner d'un renforcement purement formel des acteurs 


\section{4}

subnationaux, dans le cas où les politiques de développement restent formulées au niveau central (Aïssaoui, 2003).

Un autre angle envisageable des études sur l'élargissement consiste à se pencher sur son impact probable sur la gouvernance communautaire. Certains travaux s'intéressent au rapport entre l'adhésion d'un nombre important d'Etats à l'UE et l'avenir de l'intégration européenne (Zielonka, Mair, 2002). En réfléchissant simultanément à son impact sur les anciens et futurs Etats membres, Likke Friis et Anna Murphy soulignent le paradoxe de l'élargissement: ce processus d'extension de la gouvernance communautaire vers l'Est, qui permet de stabiliser l'environnement extérieur de l'UE, constitue aussi une menace pour son mode de gouvernement (Friis, Murphy, 1999).

L'élargissement est considéré comme une extension des frontières de l'UE à quatre niveaux : géopolitique (fin de la guerre froide), institutionnel-juridique (reprise de l'acquis communautaire par des pays non membres), transactionnel (accès régulé au marché communautaire) et culturel (normes politiques à respecter pour se rapprocher de l'UE). Entre 1989 et 1997, les frontières de l'UE deviennent plus malléables car celle-ci crée avec les pays postcommunistes un «ordre négocié » fondé sur des relations contractuelles et l'exigence de reprise de l'acquis : le lien entre adhésion formelle et gouvernance communautaire est rompu.

Friis et Murphy dégagent les logiques de ce qu'elles dénomment l'internalisation: les choix politiques en direction de l'extérieur sont déterminés par leurs conséquences attendues pour l'équilibre interne de l'Union européenne. Réciproquement, l'extension de la gouvernance communautaire à l'extérieur des frontières de l'UE influence les perspectives, les intérêts et les objectifs de l'organisation et de ses Etats membres. Les liens qui s'établissent entre les différents secteurs de politique publique affectés par l'élargissement (issue linkages) affectent les marchandages entre les Etats, et donc le développement de l'Union en termes de politiques communes, d'institutions et de budget. L'internalisation peut être considérée comme le pendant de l'européanisation des pays candidats. Cette réflexion souligne l'intégration progressive des perceptions et 
anticipations des acteurs politiques et sociaux des anciens et des futurs Etats membres de l'UE, avant même l'élargissement formel.

\section{Interrogations sur les motifs de l'élargissement}

Parmi les analyses ancrées théoriquement, un large pan de la littérature s'attache à saisir les motifs et les dynamiques de l'élargissement à travers le débat entre approches rationalistes et constructivistes. Il s'agit plus précisément d'étudier les raisons pour lesquelles les pays membres de l'UE soutiennent l'adhésion de nouveaux Etats - les motivations des pays postcommunistes pour adhérer, considérées comme allant de soi, sont souvent évoquées rapidement (Schimmelfennig, Sedelmeier, 2002).

Pour les rationalistes, deux éléments déterminent la politique communautaire à l'Est: les coûts et les bénéfices de l'élargissement pour les différents Etats membres, en termes de prospérité et de sécurité, et les rapports de force qui définissent les capacités de négociation de chacun d'entre eux. Deux types de variables entrent en jeu dans l'estimation des gains et des pertes au niveau national : des facteurs économiques (exportations croissantes vers les nouveaux membres pour les gains, concurrence dans certains secteurs économiques et perte de fonds communautaires pour les coûts) et géopolitiques (stabilisation de pays voisins, rééquilibrage de l'Union européenne vers l'Est souhaité ou redouté). Sur cette base, on distingue les « opposants » à l'élargissement, principalement les pays « de la cohésion » et du Sud de l'UE (France, Grèce, Italie, Irlande, Portugal, Espagne) et les «partisans » du Nord et de l'Est de l'Union (Autriche, Finlande, Allemagne, Danemark, Suède, Grande-Bretagne).

L'approche rationaliste permet d'expliquer l'offre d'association faite aux pays postcommunistes en 1991, en l'absence de consensus sur l'idée d'élargissement. Les «défenseurs » de l'élargissement sont en effet en minorité parmi les Douze. Ils élaborent deux stratégies pour renverser ce rapport de forces défavorable. La première consiste à donner des compensations aux « opposants »-comme le refus de la CEE d'endosser l'objectif d'élargissement dans les accords d'association, ou encore les clauses commerciales de ces accords qui sont défavorables aux pays candidats dans les secteurs "sensibles» 
comme le textile, l'agriculture ou l'acier (Mayhew, 1998). Par ailleurs, les coûts du non-élargissement sont mis en exergue à travers les menaces d'instabilité à l'Est. Les pays postcommunistes et les «défenseurs» de l'élargissement s'accommodent provisoirement de l'association, jugée préférable à l'absence de relation institutionnalisée (Moravcsik, Haggard, 1993, Moravcsik, Vachudova, 2003). Mais la redéfinition de ces premiers marchandages intergouvernementaux, qui se traduit par un passage de l'association à la perspective d'adhésion en 1993, échappe aux approches purement rationalistes. D'une part, les concessions aux pays candidats vont au-delà du " plus petit dénominateur commun» des intérêts nationaux, et ne rééquilibrent pas les coûts et les gains escomptés par les différents Etats membres. D'autre part, les transformations de l'environnement international et les garanties proposées aux «opposants» de l'élargissement sont des arguments ambigus : les «défenseurs»de l'adhésion profitent économiquement de l'association sans avoir à partager leur pouvoir politique, tandis que la perspective d'élargissement de l'OTAN peut aussi favoriser la stabilisation des pays de l'Est.

Ces approches intergouvernementalistes sont critiquées pour leur vision réductrice de l'intégration européenne (Lequesne, 1999). Elles n'offrent en outre qu'une interprétation partielle de la décision d'élargissement. La prise en compte des facteurs cognitifs, postulant que des visions du monde, des croyances et des valeurs façonnent l'idée que les acteurs concernés se font de leur identité, de leur rôle et de leurs intérêts, permet de mieux comprendre le revirement de 1993. Le point de départ de l'analyse est ici l'existence d'un ensemble de normes partagées par l'ensemble des États de l'Union, qui sont suffisamment ancrées dans l'identité ouest-européenne pour représenter une contrainte et renforcer la position des partisans de l'élargissement. Il s'agit de la «communauté de valeurs » sur laquelle a été fondée l'intégration européenne pendant la guerre froide, à savoir la démocratie libérale, l'économie de marché et la protection des libertés individuelles. Elle s'accompagnait d'une croyance en la vocation paneuropéenne de la CEE et d'un sentiment de solidarité avec le bloc de l'Est. C'est cet ensemble de normes que sous-tend le discours officiel communautaire fondé sur la «responsabilité spéciale » envers les pays postcommunistes. 
S'ils s'accordent sur le contenu de ces normes, les travaux qui se rattachent à ce courant d'analyse divergent quant aux mécanismes par lesquels les acteurs opposés à l'élargissement adhèrent au discours sur le « devoir moral » envers l'Europe de l'Est. Selon la théorie du speech act, ces valeurs sont constitutives de l'identité collective de l'UE et le simple fait de les énoncer produit des effets politiques. Après 1989, les acteurs communautaires ne font que respecter les normes énoncées dès la déclaration d'Helsinki de 1975 qui, en tant que «promesse» d'encouragement à la diffusion des normes démocratiques, définit l'identité de l'UE (Fierke, Wiener, 1999). Parmi les arguments avancés pour justifier l'élargissement, les raisons éthico-politiques prévalent donc (Sjursen, 2002).

Pour d'autres auteurs, ces normes contraignent l'ensemble des gouvernements de l'UE, qui perdraient en crédibilité et en légitimité s'ils les reniaient publiquement. Par conséquent, les «partisans » de l'élargissement peuvent exploiter les notions de responsabilité historique ou de devoir moral, tandis que les "opposants ", forcés de se placer sur le même terrain, doivent maintenir une certaine cohérence entre leurs prises de position verbales et leurs actes. C'est l'hypothèse du "piège rhétorique» avancée par Frank Schimmelfennig : un usage stratégique des arguments, dans un cadre institutionnel et normatif relativement contraignant, engendre une distorsion entre les intérêts initiaux et la décision finale (Schimmelfennig, 2001).

Une troisième approche se place au niveau méso-politique pour mettre en lumière les complexités de la prise de décision communautaire, du fait des interactions entre Etats membres et institutions communautaires, et des divergences entre les différents services de la Commission européenne (Sedelmeier, 2001). Elle montre comment l'identité collective communautaire, qui comprend la «responsabilité spéciale » envers les pays postcommunistes, agit comme une contrainte sur les opposants à l'élargissement. Une « coalition de cause » menée par la Commission européenne construit un discours qui renforce la légitimité des positions favorables à l'élargissement et stigmatise les arguments fondés sur les intérêts nationaux. Un groupe plus ou moins hétérogène se constitue alors, qui promeut des initiatives en faveur des pays candidats en présentant chaque étape de l'élargissement comme une question de principe. 
Celui-ci devient irréversible, mais le "consensus négatif» entre les Etats membres n'est pas assez fort pour imposer les réformes nécessaires à l'adhésion de nouveaux pays. Du fait de la logique cumulative qui sous-tend cette "politique composite", aucun accord profond ne se dégage sur l'avenir de l'Union européenne élargie.

Ces approches théoriques permettent de mieux comprendre un processus d'élargissement qui a rapidement été présenté comme inéluctable, tout en étant parfois mis en œuvre à contrecœur. Elles laissent cependant dans l'ombre le niveau national de gouvernement, avec la question des modes de légitimation de l'élargissement et plus largement, de l'usage de l'intégration européenne dans les jeux politiques (Jacquot, Woll, 2004).

\section{L'élargissement: regards croisés d'anciens et de nouveaux membres de l'UE}

Les recherches françaises sur l'espace politique européen ont connu un important renouvellement avec ce qu'on pourrait appeler le «tournant sociologique ». Celui-ci constitue une avancée par rapport aux débats de nature ontologique sur la nature de l'Union qui opposent les néo-fonctionnalistes aux intergouvernementalistes. Selon Andy Smith, la vue «trop aérienne» d'une partie des travaux sur l'Europe se caractérise par une insuffisante réflexivité, privilégiant la synthèse au détriment de l'analyse ; par sa lecture a-sociologique du lien social ; enfin, par une approche dichotomique et universalisante du lien public-privé (Smith, 1999). L'adoption d'une démarche inductive de sociologie politique permet en revanche, à travers la focalisation sur les pratiques au niveau individuel, de s'interroger sur la logique des institutions dans lesquelles s'inscrivent les acteurs. On s'intéresse à leurs stratégies et à leurs mobilisations lorsqu'ils sont affectés par les règles complexes du jeu institutionnel de l'UE, règles qu'ils «jouent» ou qu'ils «subissent». Qu'il s'agisse d'acteurs individuels, collectifs ou de réseaux, l'étude de «l'espace sociopolitique européen » permet de montrer comment les uns et les autres cherchent à acquérir d'autres ressources ou à renforcer leur reconnaissance, et comment leur socialisation nationale antérieure se trouve confrontée à de nouvelles pressions identitaires (Guiraudon, $2000: 18)$. 
Malgré son côté quelque peu rigide, la distinction entre les approches de l'intégration européenne "par les secteurs » et "par les acteurs », peut être transposée pour illustrer le cloisonnement des études portant sur l'élargissement de l'UE. Les perspectives dominantes s'inscrivent dans une approche sectorielle, privilégiant les acteurs institutionnels, une perspective «par le haut» et la construction des politiques européennes, alors que le renouvellement consisterait à réorienter la focale sur les acteurs (Pasquier, Weisbein, 2004). Cette démarche "par le bas» qui considère que "tous les acteurs sociaux peuvent contribuer à définir l'enjeu européen » privilégie une perspective cognitive pour s'intéresser à la manière dont ces derniers réinterprètent les politiques européennes, parfois en dehors du contexte institutionnel communautaire (id: 13). Une approche décloisonnée qui s'intéresse aux configurations des acteurs permet d'étudier la manière dont ceux-ci parviennent à politiser les enjeux européens, "à en faire un nouveau foyer de ressources et de contraintes pour l'action et notamment pour l'action publique» (Courty, Devin, 2001 : 4). Plusieurs façons de réintroduire les acteurs au centre de l'investigation se présentent. Citons les travaux sur les groupes d'intérêt qui ont cherché à influencer le processus de l'élargissement (Saurugger, 2003), les analyses de la politisation et du mode de légitimation des commissaires européens (Joana, Smith, 2002), ou encore les recherches sur la professionnalisation des «métiers de l'Europe »(Georgakakis, 2002). L’interrogation sur la manière dont les acteurs des nouveaux Etats membres modifient leurs répertoires d'action, tout en profitant d'opportunités nouvelles pour agir sur les institutions communautaires, pourrait fournir une trame à l'actualisation de recherches empiriques déjà entamées sur les mobilisations des acteurs étatiques et non-étatiques dans ces pays (Dakowska, 2002, Neumayer, 2002).

Si l'on se penche sur l'impact de l'intégration européenne sur les structures nationales, opérationnalisé par différents auteurs à l'aide du concept d'« européanisation descendante», celles-ci se sont largement concentrées sur les politiques publiques (policies) et les structures institutionnelles (polity). Les travaux sur les effets de l'intégration européenne sur les partis politiques et les compétitions partisanes (politics) demeurent rares. La grille d'analyse proposée par Klaus 
Goetz et Simon Hix est ouverte à cette dernière dimension (Goetz, Hix, 2000 : 10). Elle permet d'observer les dynamiques des relations entre les pressions européennes et les acteurs nationaux, en prenant en compte le double impact de l'intégration européenne :

1. Sous l'angle de la contrainte: dans les pays membres comme dans les Etats candidats, la modification ou l'imposition de certaines options de politique intérieure, ainsi que les répercussions des pressions de l'UE sur le système institutionnel et les relations entre les différents niveaux de pouvoir.

2. Sous l'angle de l'opportunité : la façon dont la création d'un niveau supérieur de gouvernance fournit de nouvelles ressources aux acteurs pour promouvoir des politiques publiques ou pour s'assurer des avantages informationnels. Dans le cas de l'élargissement on se penche sur la manière dont des acteurs politiques, sociaux, et économiques se saisissent de cet enjeu pour tisser des liens entre les pays candidats et les membres de l'Union.

Ainsi défini, le concept d'européanisation permet de s'interroger sur l'usage de la contrainte d'adaptation par les acteurs, en insistant sur les interdépendances entre Etats membres anciens et nouveaux. Dans cette perspective, ce dossier comprend les contributions d'auteurs qui analysent trois grands axes de l'élargissement à l'Est de l'Union européenne :

\section{Conceptualiser la politique d'élargissement}

Dans leur étude comparée des différentes vagues d'expansion de l'Union européenne, José Torreblanca et Sonia Piedrafita mettent en lumière les faiblesses des interprétations de l'élargissement actuel qui sont strictement rationalistes, c'est-à-dire fondées sur des marchandages entre des intérêts nationaux, et purement constructivistes, donc reposant sur le sentiment d'identité européenne commune et de devoir moral envers l'Europe postcommuniste. Ils plaident pour une combinaison de ces perspectives afin de montrer comment les normes qui définissent l'identité communautaire se sont combinées avec la défense des intérêts nationaux, dans un contexte de «supranationalisme délibératif» réunissant les représentants des différents Etats membres. A la lumière de cette approche théorique, 
les auteurs interprètent certains choix de la politique orientale de l'UE en montrant que celle-ci découlait non seulement d'une logique instrumentale de maximisation des intérêts, mais aussi de la volonté de prendre des décisions congruentes avec les principes fondateurs de l'UE, afin de légitimer l'adhésion de nouveaux membres.

\section{Anticiper l'adhésion}

Trois aspects complémentaires de l'anticipation de l'adhésion dans les nouveaux Etats membres constituent le second pan de ce dossier, constitué d'études de cas au niveau méso-politique.

Les acteurs institutionnels et non institutionnels des pays candidats font l'objet de l'analyse de Vello Pettai et de Viljar Veebel, à travers l'examen de la participation de différents acteurs administratifs et politiques estoniens à la Convention sur l'avenir de l'Europe en 2002-2003. En se focalisant sur les interactions entre le processus constitutionnels communautaire et les jeux politiques estoniens, ils soulignent les difficultés entourant l'élaboration d'une position nationale du fait de la succession de plusieurs échéances : la clôture des négociations d'adhésion avec l'UE, des élections législatives et une consultation référendaire. Le processus de prise de décision s'avère par conséquent fortement centralisé et marqué par l'évolution de la configuration politique au niveau national. Si l'absence d'un large débat dans la société civile pendant la tenue de la Convention ne constitue pas une particularité estonienne, cet article montre que les rapports entre les acteurs politiques internes sont essentiels à la compréhension du processus d'élargissement.

Hakim Aïssaoui se penche sur l'européanisation de l'action territoriale en Pologne, au prisme des héritages institutionnels et des enjeux de politique interne. Il montre dans sa contribution que l'adoption d'une nouvelle structure d'administration territoriale, dans la perspective de la mise en place des fonds structurels, a abouti à une décentralisation formelle qui n'a pas modifié en profondeur les pratiques de politique régionale. Les réformes administratives menées en vue de l'adhésion se sont heurtées aux luttes pour le contrôle des ressources matérielles et symboliques qui opposaient les administrations déconcentrées et décentralisées au niveau régional. Du fait des jeux politiques des responsables gouvernementaux et des 
faibles capacités d'action des collectivités locales, ces affrontements ont abouti à une centralisation de la programmation des fonds structurels, dont s'est accommodée la Commission européenne.

Enfin, Dorothée Bohle et Dora Husz présentent une double étude de cas sur le rôle des groupes d'intérêt dans les négociations d'adhésion de la Hongrie à l'Union européenne. Leur contribution critique permet de dépasser les approches focalisées sur les marchandages intergouvernementaux et l'asymétrie de pouvoir entre Etats candidats et pays membres. Ainsi, les firmes multinationales (FMN) implantées en Hongrie ont su exploiter les opportunités offertes par l'élargissement, en recourant aux différents répertoires de l'action collective afin d'obtenir des dérogations à la mise en œuvre de l'acquis communautaire en matière de politique de concurrence. Dans le cas de la libre circulation des personnes, c'est la pression syndicale et l'argument des craintes des sociétés occidentales qui sont à l'origine des périodes transitoires imposées aux nouveaux pays membres. Les auteurs montrent qu'on ne peut comprendre les logiques qui soustendent le déroulement et les résultats des négociations d'adhésion sans prendre en compte la mobilisation de puissants acteurs transnationaux.

\section{L'impact de l'élargissement sur la politique étrangère l'UE}

Pour finir, Alexandra Goujon analyse la dimension géopolitique de l'élargissement, à travers la construction de la "politique de nouveau voisinage » dirigée notamment vers les pays de l'ex-URSS dont la vocation à adhérer à l'Union européenne est controversée. Elle montre les interactions entre les institutions communautaires impliquées dans l'élaboration de cette nouvelle politique, tout en soulignant sa finalité ambiguë - puisque des ajustements sont demandés sans que la perspective d'adhésion de ces pays soit assurée. Ce papier présente en contre-point la réception de cette politique par deux de ses destinataires, l'Ukraine et la Biélorussie, qui se positionnent différemment par rapport aux demandes et aux offres de partenariat communautaire, et dont les acteurs politiques utilisent les questions européennes dans leurs jeux politiques internes. On aborde ainsi un aspect de "l'après-élargissement » qui témoigne des défis de la nouvelle Union en termes d'action internationale. 
Si l'on repose la question de l'impact de l'adhésion des nouveaux Etats membres à l'UE à la lumière des contributions qui composent ce dossier, il apparaît que le moment de l'adhésion ne bouleverse pas fondamentalement les structures administratives et politiques déjà mises en place par anticipation dans la période de pré-adhésion. En outre, l'analyse du processus d'élargissement gagne à tenir compte des mobilisations des acteurs des nouveaux Etats membres. Une perspective interactionnelle permet de mieux saisir les modalités de leur insertion dans le jeu multi-niveaux de l'Union européenne, à l'interface entre les structures nationales et communautaires. Si leurs héritages institutionnels demeurent un prisme supplémentaire d'interprétation des normes communautaires et un facteur de diversité, les acteurs et institutions des nouveaux pays membres deviennent néanmoins un objet normalisé des études européennes. Enfin, l'analyse des conséquences de l'élargissement pour l'UE, audelà d'un affinement analytique permettant de nuancer les hypothèses existantes, appelle à des études empiriques plus poussées dans les années à venir. En effet, la comparaison avec les précédentes vagues d'élargissement montre que c'est à partir de leur adhésion que les nouveaux membres de l'Union assimilent en accéléré les règles de négociation à niveaux multiples, et cherchent à tirer des bénéfices de leur nouvelle position en externalisant collectivement leurs intérêts et leurs revendications internes (Preston, 1997).

\section{BIBLIOGRAPHIE}

Ágh A. (1999), «Europeanization of Policy-Making in East Central Europe : the Hungarian Approach to EU Accession », Journal of European Public Policy, 6(5) : 839-854.

Aïssaoui H. (2003), « Partis politiques et développement territorial : le cas de la Région Silésie », in Antoine Roger (dir) Des partis pour quoi faire? La représentation politique en Europe centrale et orientale, Bruxelles, Bruylant: 173-190.

Batt J. (1997), "The International Dimension of Democratisation in Hungary, Slovakia and the Czech Republic », in Pridham G., Herring 
E., Sandforf G. (dir), Building Democracy? The International Dimension of Democratization in Eastern Europe, Londres, Leicester University Press :154-169.

Chorośnicki M., Cziomer E. (1999), Unia Europejska, transformacja i integracja w Europie (L'Union européenne, la transformation et l'intégration en Europe), Cracovie, Korona.

Courty G., Devin G. (2001), La construction européenne, Paris, La Découverte.

Cultures et Conflits (2000), "Sociologie de l'Europe. Mobilisations, élites et configurations institutionnelles », 38-39, numéro spécial.

Dakowska D. (2002), «Beyond Conditionality: EU Enlargement, European Party Federations and the Transnational Activity of German Political Foundations ", in Perspectives on European Politics and Society, 3 (2) : 271296.

Dakowska D. (2003), «Usages et mésusages du concept de gouvernance appliqué à l'élargissement de l'Union européenne », Politique européenne, $10: 99-120$.

Dimitrova A. (2002), "Enlargement, Institution-Building and the EU's Administrative Capacity Requirement », West European Politics, 25(4) : 171-190.

Fierke K., Wiener A. (1999), «Constructing Institutional Interests : EU and NATO Enlargement », Journal of European Public Policy, 6 (3): 721-742.

Friis L., Murphy A. (1999), «The European Union and Central and Eastern Europe : Governance and Boundaries ", Journal of Common Market Studies, 37(2) : 211-232.

Georgakakis D. (dir) (2002), Les métiers de l'Europe politique. Acteurs et professionnalisation de l'Union européenne, Strasbourg, Presses Universitaires de Strasbourg.

Goetz K. H., Hix S. (dir.) (2000), «Introduction: European Integration and National Policy Systems », West European Politics, dossier spécial «Europeanised Politics? European Integration and National Political Systems », $23(4): 1-26$.

Goetz K. H. (2001), «Making sense of post-communist central administration: Modernization, Europeanization or Latinization? ", Journal of European Public Policy, 8 (6) : 1032-1051.

Grabbe H., Hughes, K. (1997), The Eastwards Enlargement of the EU, Londres, Royal Institute for International Affairs.

Grabbe H. (2001), «How does Europeanization affect CEE governance? Conditionality, diffusion and diversity ", Journal of European Public Policy, 8(6): 1013-1031.

Guiraudon V. (2000), «L'espace sociopolitique européen, un champ encore en friche?", Cultures et Conflits, «Sociologie de l'Europe. Mobilisations, élites et configurations institutionnelles », 38-39 : 7-37. 
Hausner J., Marody M. (dir.) (2000), The Quality of Governance: Poland closer to the European Union ?, collection EU Monitoring IV, Varsovie, Fondation Friedrich Ebert.

Héritier A. \& al. (dir.) (2001), Differential Europe. The European Union Impact on National Policymaking, Oxford, Rowman\&Littlefield.

Ingham H., Ingham M. (2002), European Union Expansion to the East. Prospects and Problems, Cheltenham (R-U), Northampton, (E-U/MA), E. Elgar.

Inotai A. (2001), "Some Reflections on Possible Scenarios for EU Enlargement and Some Key Issues in Understanding the Negotiations on Accession to the EU », Working Papers $\mathrm{n}^{\circ} 122$, Budapest, Institut d'économie mondiale.

Jacquot S., Woll C. (dir) (2004), Les usages de l'Europe. Acteurs et transformations européennes, Paris, L'Harmattan.

Joana J., Smith A. (2002), Les commissaires européens. Technocrates, diplomates ou politiques, Paris, Presses de Sciences Po.

Kohler-Koch B., Eising R. (dir.) (2000), The Transformation of Governance, Londres, Routledge.

Lequesne C. (1999), "Quand l'Union européenne reste une affaires d'Etats », Critique internationale, 2 : 67-72.

Linden R. K. (dir.) (2002), Norms and Nannies. The Impact of International Organizations on the Central and Eastern European States, Boston, Rowman \& Littlefield.

Lippert B., Umbach G., Wessels W. (2001), «Europeanization of Central Executives: EU Membership Negotiations as a Shaping Power» Journal of European Public Policy, 8 (6): 980-1012.

Mannin M. (1999), Pushing Back the Boundaries. The European Union and Central Europe, Manchester/New-York, Manchester University Press.

Maresceau M. (1997), Enlarging the European Union, Longman, Harlow.

Mayhew A. (1998), Recreating Europe - the European Union's Policy Towards Central and Eastern Europe, Cambridge, Cambridge University Press.

Michalski A., Wallace H. (1992), The European Community: the Challenge of Enlargement, Londres, Royal Institute of International Affairs.

Moravcsik A., Haggard S. (1993), "The Political Economy of Financial Assistance to Eastern Europe 1989-1991 », in : Keohane R.O., Nye J.S., Hoffmann S.(dir), After the Cold War: International Institutions and State Strategies in Europe, Cambridge, Harvard University Press : 264285.

Moravcsik A., Vachudova M.A. (2003), « National Interests, State Power, and EU Enlargement », East European Politics and Societies, 17(1) : 4257.

Neumayer L. (2002), «La construction de l'enjeu européen dans trois pays candidats à l'adhésion à l'Union européenne : une analyse comparée 
de la Hongrie, la Pologne et la République tchèque ", Thèse de science politique, IEP de Paris.

Neumayer L. (2003), «L'européanisation indirecte des pays candidats à l'adhésion à l'Union européenne : le cas des politiques publiques de concurrence en Pologne et en République tchèque », Politique européenne, $10: 121-147$.

Neumayer L. (2004), «La science politique et l'élargissement de l'Union européenne: paradigmes et objets de recherche », dans Mink G., Forest M. (dir), Post-communisme: les sciences sociales à l'épreuve, Paris, L'Harmattan.

O'Donnell G., Schmitter P. C., Whitehead L. (1986), Transitions from Authoritarian Rule, Baltimore, John Hopkins University Press, Volume II.

Pasquier R., Weisbein J. (2004), «L'Europe au microscope du local. Manifeste pour une sociologie politique de l'intégration communautaire », Politique européenne, 12 : 5-21.

Pinder J. (1991), The European Community and Eastern Europe, Londres, Royal Institute of International Affairs.

Preston C. (1997), Enlargement and Integration in the European Union, Londres, Routledge.

Radaelli C. M. (2001), «The domestic impact of European Union public policy: notes on concepts, methods, and the challenge of empirical research », Politique européenne, $5:$ 107-142.

Raunio, T., Hix, S. (2000), «Backbenchers Learn to Fight Back: European Integration and Parliamentary Government », West European Politics, 23 (4) : 142-167.

Risse T., Börzel T. (2000), «When Europe Hits Home: Europeanization and Domestic Change », EUI Working Paper RSC : 56/2000, Florence, European University Institute.

Robert C. (2001), «La Commission européenne face à l'élargissement de l'Union à l'Est: la construction d'un leadership ", Politique européenne, $3: 38-60$.

Santiso J. (1996), «De la condition historique des transitologues en Amérique latine et en Europe centrale et orientale», Revue internationale de politique comparée, 3 (1).

Saurugger S. (2001), "L'élargissement de l'Union européenne ou la construction d'un objet politique problématisé », Politique européenne, $3: 5-12$.

Saurugger S. (2003), Européaniser les intérêts? Les groupes d'intérêt économiques et l'élargissement de l'Union européenne, Paris, L'Harmattan. 
Sedelmeier U. (2001), « Accomodation Beyond Self-Interest: Identity, Policy Paradigms, and the Limits of a Rationalist Approach to EU Policy Towards Central Europe », Politique européenne, 3 :13-37.

Sedelmeier U. (2002), "Sectoral dynamics of EU enlargement: advocacy, access and alliances in a composite policy », Journal of European Public Policy, 9(4) : 627-649.

Schimmelfennig F (2001), "The Community Trap: Liberal Norms, Rhetorical Action, and the Eastern Enlargement of the European Union ", International Organization, 55(1) : 47-80.

Schimmelfennig F., Engert S., Knobel H. (2002), « Costs, Commitments and Compliance. The Impact of EU Democratic Conditionality on European Non-Member States ", EUI Working Paper RSC, 2002/29, Florence, European University Institute.

Schimmelfennig F., Sedelmeier U. (2002) (dir), «European Enlargement: Theoretical and Comparative Approaches », Journal of European Public Policy, 9 (4), numéro spécial.

Sjursen, Helen, (2002) "Why expand: the question of legitimacy and justification in the EU's enlargement policy », Journal of Common Market Studies, 40 (3) : 491-513.

Skotnicka-Illasiewicz, E. (1993), « Poland in Europe : Suppliant, Creditor or Partner? », in The Dilemmas of European Identity, Varsovie, Fondation La Pologne en Europe : 22-25.

Smith A. (1999), "L'espace public européen' : une vue trop aérienne», Critique internationale, 2 : 169-180.

Šmejkal V., Had M., Polášková P. (1998), Česká Republika a Evropská Unie 1996-1998 (La République tchèque et l’Union européenne 19961998), Prague, Conseil des relations internationales.

Torreblanca J. I. (1998), « Overlapping Games and Cross-Cutting Coalitions in the European Union », West European Politics, 21(2) : 134-153.

Weidenfeld W. (1996), Mittel- und Osteuropa auf dem Weg zu der Europäische Union, Bericht zum Stand der Integrationsfähigkeit, Gütersloh, Verlag Bertelsmann Stiftung.

Zielonka J., Mair P. (dir) (2002), «The Enlarged European Union: Diversity and Adaptation », West European Politics, 25(2), numéro spécial. 